\title{
HISTOIRE ET PHILOSOPHIE DE LA MÉCANIQUE QUANTIQUE TRAVAUX RÉCENTS ${ }^{1}$
}

A l'égard des travaux historiques qui concernent la formation de la mécanique quantique, la France s'obstine depuis trente ans dans une certaine ignorance. La publication récente du premier volume des ceuvres d'Albert Einstein ${ }^{2}$, consacré à la contribution d'Einstein à la théorie quantique, commence sans doute à entamer cette indifférence. Mais il faut aussi disposer d'une vue générale

1. Euvres de Niels Bohr : Bohr Collected Works-Gesammelte Werke (BCW), éds Léon Rosenfeld, J. Rud Nielsen, puis Erik RÚdinger, Amsterdam, North-Holland Publishing Company, 1972..., onze vols prévus, dont huit sont parus.

Correspondance de Pauli: Wolfgang Pauli Wissenschafilicher Briefwechsel mit Bohr, Einstein, Heisenberg u.a. (PB), éds Karl von MeyenN, Armin HermanN, Victor Weisskopf, Berlin, Springer, 4 vol. prévus, dont deux sont parus : vol. I : 1919-1929 (1979), vol. II : 1930-1939 (1985).

Livres recents sur Bohr : John HENDRY, The Creation of Quantum Mechanics and the Bohr-Pauli Dialogue, Dordrecht, Reidel, 1984. Henry FolsE, The Philosophy of Niels Bohr: the Framework of Complementarity, Amsterdam, North-Holland Publ. Comp., 1985. Ulrich RöSEBERG, Niels Bohr. Leben und Werk eines Atomphysikers, Stuttgart, Wissenschaftliche Verlagsgesellschaft, 1985. John HoNNER, The Description of Nature. Niels Bohr and the Philosophy of Quantum Physics, New York, Oxford University Press, 1987. Dugald MuRDOCH, Niels Bohr's Philosophy of Physics, Cambridge, Cambridge University Press, 1987. Sandro Petruccioli, Atomi Metafore Paradossi. Niels Bohr e la costruzione di una nuova fisica, Rome, Theoria, 1988.

Sur Pauli : W. Pauli. Das Gewissen der Physik, Charles P. EnZ, Karl von MEYenN, éds, Braunschweig, Vieweg, 1988. Kalervo V. LAURIKAINEN, Beyond the Atom. The Philosophical Thought of W. Pauli, Dordrecht, Reidel, 1988.

Sur la Logique Quantique, et notamment sur le livre récent de Carl-Friedrich voN WeIzsÁcker, Aufbau der Physik, München, Carl Hanser Verlag, 1985, je ne donne ici aucune indication, mais je me permets de renvoyer à mon compte rendu dans L'Age de la Science, 2,1989, publié sous le titre « De Bohr et von Neumann à Kant ». Je signale également dans ce domaine le livre de Henry KRIPS, The Metaphysics of Quantum Theory, Oxford, Clarendon Press, 1987.

2. Albert Einstein, Euvres, Quanta, Mécanique statistique et Mécanique Quantique, Françoise Balibar, Olivier Darrigol, Bruno Jech, éds, Paris, Le Seuil, 1989.

Revue de synthèse: IV S. Nos 3-4, juil.-déc. 1989. 
de la nature et de l'étendue des problèmes posés. La question déborde d'ailleurs le domaine particulier de la philosophie de la physique, puisqu'il y a un paradoxe évident dans le fait de s'intéresser au développement de la philosophie des sciences américaines sans se donner les moyens de percevoir avec précision certaines des questions dont elle est sortie et qui sont au fondement de débats actuels sur le " réalisme " que la philosophie continentale a quelquefois du mal à situer. L'objet de cette chronique est donc à la fois de donner une idée de l'évolution de la bibliographie étrangère sur la mécanique quantique, de mentionner quelques travaux récents importants - à la fois dans le domaine de l'édition des sources primaires et dans celui des orientations de la littérature secondaire - et de rappeler les traits caractéristiques de la situation française dans ce domaine.

\section{COMPRENDRE LA FORMATION DE LA MECANIQUE QUANTIQUE}

Cette question simple, qui centre les recherches sur le processus de genèse de la théorie quantique sous la forme qu'elle prend en 1927 , oriente dans une large mesure l'évolution de la bibliographie quantique. Elle s'impose à un certain nombre d'historiens, américains pour la plupart, à partir du début des années 1960 , et les entraîne dans une direction opposée à celle que privilégiaient beaucoup d'épistémologues, surtout préoccupés de discuter des présupposés de l'interprétation dite orthodoxe de la mécanique quantique. Il est amusant de remarquer que, s'il existe actuellement un renouveau d'une réflexion philosophique sur la théorie quantique mieux dégagée du poids des préoccupations partisanes, cela est dû pour une large part à des travaux historiques qui ont revendiqué de manière parfois militante leur neutralité épistémologique. Mais si ces travaux historiques ont eu une telle importance, c'est bien parce que la formation de la mécanique quantique était envisagée jusqu'alors comme un événement compact peu problématique, isolé de ce qui l'avait rendu possible (la première théorie quantique) comme d'ailleurs de ce qui l'avait suivi presque immédiatement (la théorie quantique des champs). La rapidité de sa formation, l'intensité des relations et des polémiques, le rôle joué par une analyse des concepts fondamentaux indissociable des progrès techniques, et surtout une certaine théatralité spectaculaire dans les interactions des six ou sept personnages principaux, ont longtemps contribué à produire cet effet d'écran. Rappelons brièvement pourquoi.

En 1922, invité par David Hilbert, Max Born et James Franck, Bohr donne à Göttingen une série de conférences sur l'atome dont la tension oxymorique fait sur les auditeurs, parmi lesquels on compte Pauli et Heisenberg, une impression décisive :

« L'état actuel de la physique atomique, dit-il, est caractérisé par ceci que nous sommes convaincus d'avoir une certaine connaissance des éléments constitutifs de l'atome, et que nous sommes également convaincus qu'il n'est 
pas possible de progresser plus avant à l'aide des images de l'électrodynamique classique ${ }^{3}$.

Heisenberg a longuement commenté l'influence de ces conférences, véritable point de départ de la physique atomique en Allemagne : Bohr rendait visible un abîme. D'un côté, il exposait sa théorie du système périodique des éléments théorie confirmée cette même année 1922 dans une de ses prédictions, par la mise en évidence de l'existence de l'élément 72 [le « Hafnium »] par G. de Hevesy et D. Coster à Copenhague -, de l'autre il faisait voir toute l'étendue des difficultés de la constitution d'une dynamique atomique. A la suite de ces conférences, le séminaire de physique théorique de Göttingen s'oriente presque exclusivement dans la direction de la physique atomique - une discipline relativement nouvelle pour Max Born -, tandis que Pauli, Heisenberg et Jordan deviennent en alternance les assistants de Born et de Bohr.

Cinq années plus tard, en 1927 , le cinquième Congrès Solvay rassemble à Bruxelles tous les fondateurs de la première et de la seconde théorie quantique : Einstein et Planck, de Broglie et Schrödinger, et tous les membres (sauf Jordan) de ce qu'il était convenu depuis 1922 d'appeler le groupe de CopenhagueGöttingen : Bohr, Heisenberg, Pauli, Born, Kramers, Dirac. Le 3 novembre, Paul Ehrenfest écrit à ses étudiants Goudsmit, Uhlenbeck et Diecke une longue lettre qui commence par la description suivante :

« Bruxelles a été sensationnel! Bohr a dominé littéralement tout le monde. Il n'était absolument pas compris au début, et puis il a peu à peu remporté la victoire sur tous. Naturellement on a eu droit de nouveau aux épouvantables incantations de sa terminologie. Impossible à résumer pour qui que ce soit - le pauvre Lorentz : interprète entre les Anglais et les Français qui ne se comprennent pas. Et de résumer Bohr. Et Bohr de réagir avec un désespoir courtois. Chaque nuit, Bohr venait me voir à une heure dans ma chambre pour me dire juste trois mots, ... jusqu'à trois heures du matin. Cela a été extraordinaire pour moi d'assister aux conversations entre Bohr et Einstein. Une partie d'échecs. Einstein avec des exemples toujours nouveaux. Une sorte de mouvement perpétuel pour arriver à percer une brèche dans la relation d'incertitude. Bohr sortant d'un épais nuage de fumées philosophiques et choisissant ses instruments pour mettre en pièces chaque exemple l'un après l'autre. Einstein comme un diable dans une boîte : surgissant de nouveau chaque matin frais et dispos. C'était impayable. Mais je suis pratiquement sans réserves pro Bohr contra Einstein. Einstein se comporte à l'égard de Bohr exactement de la même manière que les partisans de la simultanéité absolue se comportaient à son égard à lui " ${ }^{4}$.

Bruxelles marque ainsi de façon très dramatique la conviction que certains problèmes conceptuels fondamentaux de la physique quantique ont été résolus.

3. D'après les notes prises par R. MINkowski ( $A H Q P$, Mf 3, $B C W 4$, p. 341-421) (traduit par moi).

4. Lettre de Paul Ehrenfest à Samuel Goudsmit, George Uhlenbeck et George H. Diecke, $B C W 6$, p. $415-418$ (traduite par moi). 
Que cette conviction soit toute récente - le principe d'indétermination n'est formulé que quelques mois avant le Congrès Solvay - , qu'il importe de la comprendre en rapport avec la décennie précédente de "désespoirs » et de tâtonnements dans l'évolution des idées sur la structure de l'atome et le mouvement des électrons, cela pèse peu en regard de l'unité conceptuelle nouvellement obtenue. La formulation par Bohr, Born, Heisenberg, Pauli, Dirac et Jordan d'une interprétation cohérente de la synthèse entre la mécanique matricielle de 1925 et la mécanique ondulatoire élaborée par Schrödinger en 1926 est si spectaculaire qu'elle a fait oublier (aux autres) le détail de ce qui s'était passé au cours de ces cinq années, entre les conférences de Göttingen et le Congrès Solvay, et dans la période précédente.

C'est ce détail que certains historiens se déterminent, aux environs de 1960 , à retrouver et à recomposer. Comprendre l'émergence de la mécanique quantique à partir des problèmes ouverts depuis la fin du XIX ${ }^{e}$ siècle par la spectroscopie; comprendre les sources de la méthodologie caractéristique de la mécanique matricielle, celle de la restriction aux observables; comprendre les raisonnements inattendus qui conduisent en 1925 Pauli à l'énoncé du principe d'exclusion, Heisenberg à réinventer le calcul matriciel, Uhlenbeck et Goudsmit à l'hypothèse du spin : de bloc compact, la formation de la mécanique quantique devenait une sorte de labyrinthe. On peut distinguer d'abord deux périodes dans cette transformation.

La première période va jusqu'au début des années 1970 . C'est celle de la constitution des Archives de la physique quantique, de la publication sensiblement concomitante d'un certain nombre d'cuvres scientifiques, de " reprints " et de correspondances, puis de la parution d'ouvrages généraux dont certains sont encore aujourd'hui des textes de référence. Le premier projet était un travail de titan; grâce à lui, aucune autre période de l'histoire de la physique ou des mathématiques ne dispose d'un corpus catalogué aussi étendu et aussi riche. Lancé en 1962 par Thomas S. Kuhn, John L. Heilbron et Paul Forman avec la collaboration de Lini Allen, le programme des Archives for the History of Quantum Physics (AHQP) comprend actuellement, outre toute la correspondance scientifique de Bohr (BSC), 79 microfilms dans lesquels on trouve l'essentiel des manuscrits, conférences et correspondances de Dirac, Sommerfeld, Schrödinger, Kramers, Goudsmit, Kemble, Fermi, etc. Il s'y ajoute un ensemble d'entretiens menés avec plus de soixante physiciens ayant pris part à la formation de la mécanique quantique, entretiens dont l'intérêt est inégal mais souvent considérable - c'est le cas en particulier de l'interview de Heisenberg par T.S. Kuhn, qui occupe plusieurs centaines de pages dactylographiées. A la même époque, on se préoccupe de rassembler les articles originaux, soit dans les Dokumente der Naturwissenschaft de Stuttgart (1962-1963), soit sous la forme de la "collection" (non publiée) constituée par Gerald Holton à Harvard, soit enfin sous celle des Sources of Quantum Mechanics ${ }^{5}$ éditées par B. L. Van der Waerden à la suite d'un

5. Sources of Quantum Mechanics, Barteel L. VAN DER WAERDEN, éd., Amsterdam, North-Holland, 1967; New York, Dover, 1968. 
travail historique remarquable dont les lettres conservees dans les $A H Q P$ gardent une trace plus précise peut-être que ne l'indique l'introduction du livre. D'autre part, K. Pzribram publie sous le titre de Briefe zur Wellenmechanik ${ }^{6}$ une sélection de la correspondance échangée entre Schrödinger, Planck, Lorentz et Einstein au moment de la formulation de la mécanique ondulatoire; traduite par M.J. Klein en anglais, cette sélection doit faire l'objet prochainement d'une réédition considérablement augmentée, notamment par l'addition des lettres de Pauli et de celles de Bohr, grâce à K. von Meyenn. Toujours entre 1960 et 1965 environ, on publie les Collected Scientific Papers de Fermi, Ehrenfest, Kramers, J. von Neumann, Pauli et Planck. A la fin des années 1960, paraissent la Correspondance d'Einstein et Besso (éditée par P. Speziali), celle d'Einstein et Sommerfeld (éditée par A. Hermann) et celle d'Einstein et Born (celle-ci sans aucun travail critique). Enfin, plusieurs ouvrages de synthèse sont publiés dès cette époque; le livre de A. Petersen sur mécanique quantique et tradition philosophique (1963), celui de K. Meyer-Abich sur la Complèmentarité de Bohr (1965), les livres de P. Heelan (1965) et de H. Horz (1966) sur Heisenberg, enfin le premier livre de M. Jammer sur la mécanique quantique, Conceptual Foundations of Quantum Mechanics (1966), qui donne une vue à la fois globale et extrêmement précise du développement de la théorie et qui reste une référence essentielle. Il faut encore mentionner les premiers articles historiques importants, ceux de Léon Rosenfeld sur Bohr notamment celui écrit en collaboration avec Erik Rüdinger sur la trilogie de 1913 $(1967)^{7}$ —, ceux de M.J. Klein - particulièrement celui de 1970 sur la « première phase du dialogue entre Bohr et Einstein ${ }^{8}$-, et des articles d'orientation plus épistémologique : ceux de A. Shimony ${ }^{9}$, de G. Holton - sur la complémentarité $(1970)^{10}$ - et de N. R. Hanson - sur l'Interprétation de Copenhague (1959) ${ }^{11}$.

La seconde période est celle des années 1970-1980, au cours desquelles sont publiées un nombre impressionnant d'études ponctuelles de très grande qualité, dont la méthodologie s'apparente pour nous à la tradition Koyréenne. En 1969 parait le premier volume de la revue Historical Studies in the Physical Sciences, fondée par Russell McCormmach et dirigée ensuite par John Heilbron. En se donnant comme objet exclusif le développement des sciences physiques dans la période "postérieure à la révolution scientifique " et "l'application des techni-

6. Schrödinger-Planck-Einstein-Lorentz : Briefe zur Wellenmechanik, Karl PzRIBRAM, éd,, Vienne, Springer, 1963. Traduction anglaise par Martin J. KLEIN, Letters on Wave Mechanics, New York, Philosophical Library, 1967.

7. L. Rosenfeld, E. RÚdinger, « The Decisive Years, 1911-1918 ", in Samuel Rozental, éd., Niels Bohr. His Life and Work as Seen by his Friends and Colleagues, Amsterdam, North-Holland, 1967.

8. M.J. KLEIN, "The First Phase of the Bohr-Einstein Dialogue ", Historical Studies in the Physical Sciences (HSPS), 2, 1970, p. 1-39.

9. Abner ShImony, "Quantum Physics and the Philosophy of Whitehead", in Boston Studies in the Philosophy of Science, vol. 2, New York, Humanities Press, 1965, p. 307-343.

10. Gerald Holton, "The Roots of Complementarity ", Daedalus, 99, 1970, p. $1015-$ 1055. Traduction française in L'Imagination scientifique, Paris, Gallimard, 1981.

11. Norman R. HANSON, "Copenhagen Interpretation of Quantum Theory ", American Journal of Physics, 27, 1959, p. 1-15. 
ques de l'histoire intellectuelle aux problèmes de la période moderne ", en refusant de publier « des articles tombant dans des catégories périmées comme la biographie héroïque, l'histoire de l'enchaînement des découvertes ou l'identification des précurseurs ", cette revue a joué un rôle extraordinairement important aux États-Unis. Parmi les ensembles d'articles les plus remarquables qui y sont publiés entre 1969 et 1980 sur la théorie quantique, on peut en citer deux. Un premier groupe concerne l'histoire de la théorie atomique de Bohr de 1913 à 1924 : l'étude par J. Heilbron et T. Kuhn des grands articles de $1913(1969)^{12}$, les articles de D. Serwer (1977) ${ }^{13}$ sur la question de la " contrainte de liaison non mécanique " entre 1923 et 1925 , de P. Forman (1970 et 1971$)^{14}$ sur l'effet Zeeman et sur la question de la causalité dans l'Allemagne de Weimar, enfin les articles de D. Cassidy (1979) ${ }^{15}$ et de E. McKinnon (1977) ${ }^{16}$ sur le premier "modèle " de Heisenberg [le " core model "]. Un second groupe d'articles proéminent dans la littérature secondaire de cette période porte sur Schrödinger. On citera l'étude du rapport entre Schrödinger et de Broglie par V. Raman et P. Forman (1969) ${ }^{17}$, et les articles de P. Hanle sur Exner et Schrödinger $(1977)^{18}$, de Linda Wessels $(1979)^{19}$ sur la genèse de la démarche de Schrödinger et de $\mathrm{H}$. Kragh $(1979)^{20}$ sur les débuts de la mécanique ondulatoire. Après l'encerclement par les $A H Q P$, toutes ces études favorisaient la précision et la rigueur et parvenaient à reconstituer la genèse des problèmes physiques et conceptuels à partir d'une utilisation de plus en plus systématique des sources primaires. En même temps, l'Office for the History and Technology de l'Université de Berkeley commençait la publication de bibliographies (Planck, Moseley, Heisenberg). Peu de livres à caractère général sont publiés pendant cette période - il faut pourtant mentionner le livre de R. Stuewer sur l'effet Compton (1975) ${ }^{21}$ -, comme si toute l'activité des chercheurs s'était concentrée sur ces opérations minutieuses de repérage cartographique.

12. John L. HeIlbron, Thomas S. KuHN, « The Genesis of the Bohr Atom ", HSPS, I, 1969, p. 211-290.

13. Daniel Serwer, "Unmechanischer Zwang : Pauli, Heisenberg, and the Rejection of the Mechanical Atom, 1923-1925 », HSPS, 8, 1977, p. 189-256.

14. Paul Forman, "Alfred Landé and the Anomalous Zeeman Effect, 19-1921 », HSPS, 2, 1970, p. 153-261, et "Weimar Culture, Causality and Quantum Theory, 1918-1927 », HSPS, 3, 1971, p. 1-116.

15. David CASsidy, " Heisenberg's First Core Model of the Atom : the Formation of a Professional Style ", HSPS, 10, 1979, p. 123-186.

16. Edward McKinnon, " Heisenberg, Models and the Rise of Matrix Mechanics », HSPS, 8, 1977, p. 137-188.

17. V.V. Raman, P. Forman, « Why Was it Schrödinger who Developed de Broglie's Ideas? ", HSPS, 1,1969 , p. 291-314.

18. P.A. HANLE, "Indeterminacy before Heisenberg : the Case of Franz Exner and Erwin Schrödinger ", HSPS, 10, 1979, p. 225-270.

19. Linda WESSELS, "Schrödinger's Route to Wave Mechanics ", Studies in the History and Philosophy of Sciences, 10, 1979, p. 311-340.

20. Helge KRAGH, "On the History of Early Wave Mechanics ", IMFUFA tekst, 23, 1979 (Roskilde University Center, Danemark) et « Erwin Schrödinger and the Wave Equation : the Crucial Phase ", Centaurus, 26, 1982, p. 154-197.

21. Roger Stuewer, The Compton Effect. A Turning Point in Physics, New York, 1975. 
Centrée sur la formation de la mécanique quantique entre 1922 et 1927 , la recherche historique s'est donc attachée, depuis 1960, à deux tâches principales, d'une part l'établissement des archives et la mise en place de repères généraux, d'autre part l'accumulation d'analyses ponctuelles très minutieuses ordonnées selon deux ou trois axes principaux. Certaines questions, en revanche, n'ont encore reçu aucun traitement systématique, comme celle des rapports entre mathématiciens et physiciens dans l'Allemagne des années 1930.

1980-1988 : ÉDITIONS RÉCENTES MAJEURES ET PRINCIPALES ORIENTATIONS

Sans doute n'est-il pas exagéré de voir depuis 1980 une nouvelle modification de l'orientation des travaux sur la mécanique quantique. Marquée à l'extérieur par la publication de plusieurs livres importants sur Bohr et Pauli ${ }^{22}$, cette modification tient de manière moins évidente à un intérêt croissant pour la signification philosophique d'une histoire conceptuelle qui continue, par ailleurs, à bénéficier de l'habitude de la rigueur qu'elle a acquise dans les années 1970. Aussi paradoxal que cela puisse paraitre si l'on envisage l'énormité de la bibliographie concernant Bohr, le sentiment qui s'est imposé depuis quelque temps est que l'essentiel reste à faire dans le domaine d'une interprétation adéquate de ses idées. Selon une remarque récente de $\mathrm{J}$. Cushing,

"peut-être est-il temps pour les philosophes des sciences de s'efforcer de prendre au sérieux les idées philosophiques de Bohr concernant la complémentarité. Ce n'est que tout à fait récemment que quelques philosophes P. Teller, H. Folse, D. Howard - ont tenté de présenter la philosophie de Bohr comme un tout cohérent, au lieu d'y voir un ensemble contradictoire de positions philosophiques naives ${ }^{23}$.

La même observation s'applique indiscutablement à Heisenberg : si la notion de complémentarité a dû à sa difficulté d'être mal comprise ou faussement interprétée, que dire des idées de Heisenberg sinon qu'elles ont été à la fois enfouies et pillées, et que peu de gens se préoccupent actuellement de savoir pourquoi l'interprétation de la mécanique quantique a poussé le physicienmusicien-helléniste qu'il était à développer le concept de style à propos des théories physiques ou à écrire tant d'articles sur les Grecs. Même observation, dira-t-on, pour Schrödinger, plus maltraité encore puisqu'il n'existe aucune étude d'ensemble de son énorme production philosophique, qu'il concevait du reste

22. Voir note 1.

23. James Cushing, "Foundational Problems in and Methodological Lessons from Quantum Field Theory ", in Harvey R. BROWN, Rom HARRE, Philosophical Foundations of Quantum Field Theory, Oxford, Clarendon Press, 1988, p. 29. 
comme tout à fait indépendante de son cuvre de physicien ${ }^{24}$. Même observation, enfin, pour Pauli - jusqu'à présent la seule tentative de restitution de l'unité de ses idées philosophiques se trouve dans un article de Heisenberg (Die Naturwissenschaften, 24, 1959, p. 661-663) -, pour Max Born et pour Pascual Jordan. Aussi les orientations philosophiques des fondateurs de la mécanique quantique - qu'on les prenne isolément ou en groupe, fondus alors dans la désignation collective de l' "Interprétation de Copenhague " - sont-elles encore très mal connues.

La mise en évidence progressive de cette constatation a eu pour effet un renouveau très important des travaux sur Bohr et Pauli. Pourquoi Bohr et Pauli sont-ils pour le moment les premiers bénéficaires de cette nouvelle orientation? La raison la plus immédiate en est l'influence de la publication, encore inachevée, d'une part des œuvres de Bohr, d'autre part de la correspondance scientifique de Pauli (voir note 1). Dans les deux cas, ces projets se heurtaient à toutes sortes de difficultés. S'agissant de Bohr, il fallait classer et cataloguer son immense correspondance, traduire en anglais tous les textes écrits en danois et la plupart de ceux écrits en allemand, dépouiller les manuscrits très nombreux, effets d'incessantes réécritures, introduire et annoter chacun des volumes. S'agissant de Pauli, il fallait non seulement retrouver les lettres -2000 ont été ainsi rassemblées -, mais également interpréter les innombrables éléments nouveaux qu'elles apportaient à la connaissance de la formation de la mécanique quantique. Dans les deux cas, la solution patiente et rigoureuse donnée à ces difficultés a fait de ces deux éditions les références indispensables de tout travail historique ultérieur.

L'édition des Bohr Collected Works (BCW) avait comme premiers « éditeurs généraux " Léon Rosenfeld et J. Rud Nielsen, et pour certains volumes Rudolph Peierls, Jens Thorsen et Ubrich Hoyer; elle est dirigée depuis 1984 par Erik Rüdinger, avec la collaboration de Jorgen Kalckhar, Klaus Stolzenburg et David Favrholdt. Commencée en 1972, après avoir connu certains changements d'orientation, cette édition est maintenant presque achevée, puisque sur onze volumes prévus, huit sont parus ; le volume 10, consacré aux textes philosophiques de Bohr après 1928, est sous presse, et si le volume 7 est encore inachevé, la cause en est due aux difficultés légendaires de l'interprétation de l'article de Bohr-Rosenfeld de 1933. Les 4500 pages publiées à ce jour associent de manière à la fois thématique et chronologique les textes, les manuscrits et l'essentiel des lettres, dans un effort de présentation et d'annotation qui en rend l'utilisation très facile, particulièrement dans le cas du volume 5 (1924-1926) paru en 1984 et remarquablement introduit par $\mathrm{K}$. Stolzenburg et $\mathrm{E}$. Rüdinger. L'édition des $B C W$ a permis de révéler la continuité de la pensée de Bohr entre 1913 et 1939. Un exemple en est la question de l'interprétation du "principe de correspondance ", qui intéresse plus généralement l'épistémologie puisqu'il s'agit de la situation de la théorie quantique par rapport à la physique classique : on en suit les differentes

24. Ce point est mis en évidence dans l'article de Michel BrnBol, « Mach et Schrödinger : de l'élément au tout ", à paraitre, Fundamenta Scientiae, 1989. Voir aussi la traduction par M. BrrBoL, de Mind and Matter, à paraître au Seuil, 1989. 
formulations depuis les conjectures de 1914 jusqu'à l'idée énoncée en 1919 d'un accord asymptotique de la théorie atomique avec la théorie classique pour les grands nombres quantiques, et jusqu'à la persistance de «considérations de correspondance " non seulement dans la théorie de la dispersion de KramersHeisenberg (1924), mais même dans la mécanique matricielle de Heisenberg (1925). Les volumes 5 et 6 permettent ainsi de comprendre la manière dont le principe de correspondance se transforme progressivement dans l'idée d'une "complémentarité de la description ", c'est-à-dire dans l'idée que la spécificité quantique tient à la mutuelle exclusion des possibilités de définition et des possibilités d'observation de l'objet. Cette idée sert, à partir de 1927, à résoudre les contradictions dans l'interprétation du formalisme que la controverse de 1926 avec Schrödinger avait mises en pleine lumière. Enfin, la correspondance de Bohr éclaire de manière essentielle la genèse de ses idées, par exemple sur la question du principe de conservation stricte de l'énergie et de l'impulsion (lettres à C. G. Darwin et à J. Franck, notamment), sur l'idée de champ « virtuel ", sur le rôle des images, sur le langage "intuitif" de la physique classique, etc. Elle témoigne en même temps de ses réactions à chaque nouvelle tentative de solution des difficultés de la théorie atomique, par exemple de son adhésion immédiate, malgré le scepticisme de Pauli et de Heisenberg, à l'idée du spin de l'électron timidement proposée en 1925 par S. Goudsmit et G. Uhlenbeck, alors que ce dernier s'apprêtait à abandonner la physique pour les lettres classiques.

L'édition de la correspondance de Pauli, d'autre part, est l'œuvre de Karl von Meyenn, Armin Hermann et Victor Weisskopf; les Scientific Papers de Pauli avaient déjà été publiés par R. L. Kronig et V. Weisskopf en 1964, de même que ses célèbres Cours de physique, édités par Ch. Enz en 1973. Les deux volumes parus (sur quatre de la Correspondance) ont néanmoins eu un grand retentissement, non seulement parce qu'il est difficile de concevoir un travail d'annotation historique et critique plus impeccable que celui qu'ont accompli ici les éditeurs, mais aussi parce que l'influence de Pauli sur la formation de la mécanique quantique s'y révèle bien plus grande qu'on ne l'avait cru jusqu'alors. Publiant peu, et seulement ce qu'il estimait avoir démontré, Pauli ne s'était pas encore vu reconnaître le rôle décisif qu'on lui accorde maintenant. La raison principale de la situation paradoxale qui était la sienne dans la tradition orale est sans doute à chercher dans le poids et le mystère de sa personnalité : on raconte toujours certaines de ses colères, on rappelle l'efficacité du "Pauli-effekt» (la seule présence de Pauli était réputée suffire à dérégler le comportement mécanique de tous les instruments environnants), on se souvient éventuellement de sa connaissance de Goethe et du rôle de Méphistophélès qu'il jouait dans la parodie du Faust montée à l'Institut Bohr en 1932, on s'interroge sur la façon dont il a pu, à partir d'harmonies numériques, formuler le principe d'exclusion, on relit avec surprise l'excellente analyse de Kepler qu'il a écrite pour le livre signé avec « son » psychanalyste, Carl Jung. Mais seule la publication de la Correspondance pouvait donner une réelle connaissance de son travail scientifique, épuré dans ses articles ; c'est, en effet, dans ses lettres qu'il discute longuement de ses idées et de celles des autres, rapportant par exemple à Bohr les objections d'Einstein à l'égard de la théorie du champ virtuel de 1924, et encourageant Heisenberg dans 
la direction de la redéfinition des concepts fondamentaux de la cinématique, dont sort la mécanique matricielle. Qu'il s'agisse de l'abandon des modèles et des images de la physique classique (celle de trajectoire, notamment) ou de la formulation du principe d'exclusion, du principe de restriction aux observables (formulé par lui dès 1921) ou du calcul des intensités pour l'atome d'hydrogène (première « application » réussie du formalisme matriciel), Pauli mêlait constamment au travail technique l'analyse des concepts fondamentaux et son influence décisive sur le développement de la théorie quantique doit donc à présent être envisagée de manière plus globale.

Faisant usage de ces deux éditions et des travaux en cours concernant les sources primaires - particulièrement, ceux de Helmut Rechenberg pour l'édition des ceuvres de Heisenberg ${ }^{25}-$, plusieurs livres très récents renouvellent la discussion des idées philosophiques de Bohr et de l'évolution conceptuelle qui a conduit à la mécanique quantique. Dans l'impossibilité de donner ici un compte rendu détaillé de chacun de ses livres, et de leurs divergences d'interprétation, je me limiterai à souligner leurs points communs les plus évidents : un usage systématique des $A H Q P$, et particulièrement des correspondances scientifiques; un rejet des idées reçues et de la glose habituelle au sujet de l'Interprétation de Copenhague; une attention nouvelle à la précision du vocabulaire. Ainsi, la référence aux lettres échangées notamment entre Bohr et Pauli entre 1922 et 1928 permet-elle à J. Hendry d'établir l'importance de l'influence du second sur la création de la mécanique quantique. Le rejet des idées toutes faites sur Bohr et l'esprit de Copenhague - idées particulièrement à la mode en France - permet à $\mathrm{J}$. Honner ou à $\mathrm{D}$. Murdoch de faire justice du curieux reproche de subjectivisme et de positivisme que l'on trouve, par exemple, dans les écrits de M. Bunge ou de K. Popper, et qu'aucun texte ne justifie. L'attention au vocabulaire de Bohr et de Pauli - dont il faut une fois pour toutes attribuer le premier mérite à E. Scheibe ${ }^{26}$ - permet de débrouiller les usages de termes comme " mysticisme ", " symbolismes ", " images », etc., dont le moins qu'on puisse dire est que leur interprétation précipitée avait littéralement créé à partir de rien la florissante industrie de la nouvelle gnose quantique. En ce qui concerne plus spécialement les livres de H. Folse et de J. Honner enfin, il faut mentionner que leur confrontation donne une idée beaucoup plus claire que précédemment du rapport qu'il est raisonnable d'établir entre Bohr et Kant. Si le terme de «transcendantal », présent dans le titre du livre de J. Honner, sert à insister sur l'existence chez Bohr d'une explication des conditions de possibilité de l'expérience en général, J. Honner reconnaît avec $\mathrm{H}$. Folse que le déplacement opéré par Bohr en direction du problème de la communication de l'expérience dans le discours a une signification philosophique importante, bien qu'encore non éclaircie.

25. Signalons notamment la récente publication d'un manuscrit philosophique de Heisenberg intitulé « Was ist eigentlich Wirklichkeit? » grâce aux soins de Helmut RECHENBERG, à Munich, chez R. Piper.

26. Erhard SCHEIBE, The Logical Analysis of Quantum Mechanics, Dordrecht, Reidel, 1973, chap. 1 . 
Il est impossible de clore cette présentation sommaire des principales orientations actuelles sans mentionner le travail de $M$. Redhead et du séminaire de philosophie de la physique de l'Université d'Oxford. Ce séminaire vient de publier un recueil d'articles intitulé Philosophical Foundations of Quantum Field Theory ${ }^{27}$, dont l'objet est de marquer l'intérêt spécifique des problèmes de fondement de la théorie quantique des champs. Sur la question de l'existence de cette spécificité, les avis sont d'ailleurs partagés, certains pensant que cette théorie ne fait que poser dans un formalisme mathématique beaucoup plus compliqué les mêmes problèmes fondamentaux que la mécanique quantique non relativiste. Le livre commence avec un article de $\mathbf{M}$. Redhead, " $\mathbf{A}$ Philosopher Looks at Quantum Field Theory ", qui reprend le développement que l'auteur avait déjà donné en 1982 de l'affirmation suivante de $\mathrm{H}$. Stein : « La théorie quantique des champs est le lieu contemporain de la recherche métaphysique. " Les autres contributions concernent les problèmes des particules virtuelles et de la renormalisation, de la théorie de jauge et de l'axiomatisation.

Au vu de cette évolution récente des recherches historiques et philosophiques sur la mécanique quantique, on ne peut que constater la vie et l'animation qui y règnent. Une difficulté bien connue de la philosophie est d'avoir un problème suffisamment en main pour que l'on puisse s'essayer à le formuler. Après soixante ans d'existence de la mécanique quantique, il semble que certaines questions fondamentales commencent à prendre forme.

\section{LA SITUATION DE LA FRANCE}

Que dire pourtant de la situation de la France?

En 1922, un des meilleurs exposés qui existent de la première théorie atomique de Bohr paraît en français, celui de E. Bauer, La Théorie de Bohr. La constitution de l'atome et la classification périodique des éléments ${ }^{28}$. En 1923, on pouvait lire la traduction française des Drei Aufsätze de Bohr (1922), sous le titre de Les Spectres et la structure de l'atome ${ }^{29}$; en 1924, celle du complément ajouté en 1921 par N.R. Cambell à son livre Physics, The Elements, sous le titre de Théorie quantique des Spectres ${ }^{30}$; en 1932 , l'on disposait de la traduction du premier grand recueil d'articles de Bohr (1931), sous le titre de La Théorie atomique et la description des phénomènes ${ }^{31}$, en 1932 également, A. Proca traduisait (avec J. Ullmo) les Principes de la mécanique quantique ${ }^{32}$ de Dirac, publiés en anglais

27. Voir note 3, p. 7. Contributions de M. Redhead, J. Cushing, R. Weingard, R. Harré, P. Teller, G. Fleming, T. Y. Cao, R. Streater, S. Saunders.

28. Paris, Hermann, 1922.

29. Paris, Hermann, 1923.

30. Paris, Hermann, 1924.

31. Paris, Gauthier-Villars, 1932.

32. Paris, P.U.F., 1931. 
en 1930, puis, en 1933, les Mémoires sur la mécanique ondulatoire de Schrödinger ${ }^{33}$, publiés en allemand en 1927. Ce n'est pas tout : les Congrès Solvay étaient naturellement publiés en français - celui de 1921 : Atomes et Electrons, celui de 1927 : Electrons et Photons ${ }^{34}$, etc. Les "Gifford Lectures" d'A. Eddington de 1927, qui donnent un exposé synthétique extraordinairement clair de la nouvelle mécanique, sont traduites en 1929 sous le titre de La Nature du monde physique 35 . Un nombre important d'ouvrages collectifs paraissaient régulièrement, parmi lesquels il faut mentionner les Semaines de synthèse. La collection des Actualités scientifiques et industrielles, fondée par Freyman aux éditions Hermann, traduisait dans un délai très court, souvent sous forme de monographies, la plupart des textes importants de philosophie de la physique et des mathématiques qui paraissaient en Allemagne ou en Italie. La revue de A. Koyré, H.-Ch. Puech et A. Spaier, Recherches philosophiques, qui paraît de 1932 à 1938, publiait également de nombreux textes dans $œ$ domaine. Citons, enfin, l'activité d'A. George et de M. Boll.

Ensuite, l'édition s'essouffle et les choix se portent presque exclusivement sur les textes de caractère général, au détriment des textes techniques et des sources primaires. Même en ce qui concerne le domaine des premiers, les traductions ne sont pas assez nombreuses et systématiques pour être représentatives. Le second recueil d'articles de Bohr (1958) est traduit en 1961, mais pas le troisième, Essays 1958-1962 on Atomic Physics and Human Knowledge ${ }^{36}$. Les " Gifford Lectures " de Heisenberg (1955) sont traduites également en 1961 - c'est Physique et Philosophie - et son autobiographie intellectuelle (1969) parait en français en 1972 - La Partie et le Tout. Mais on ne dispose toujours d'aucune traduction des trois autres recueils d'articles de Heisenberg : Wandlungen in den Grundlagen der Naturwissenschaft ${ }^{37}$ (1935 et 1949), Schritte über Grenzen ${ }^{38}$ (1971) et Tradition in der Wissenschafi ${ }^{39}$ (1977). En ce qui concerne les textes plus techniques écrits par Bohr et Heisenberg, ou les écrits, quels qu'ils soient, de Schrödinger, Born, Jordan, Dirac, Pauli, on avoisine le néant. Même chose en ce qui concerne des textes dont on pourrait penser qu'ils sont à l'évidence proches de la tradition " continentale », comme le Philosophy of Mathematics and Natural Science de H. Weyl (1927 et 1949) ou le Determinism and Indeterminism in Modern Physics de E. Cassirer (1936).

" Dans l'Orient désert, quel devint mon ennui..." "

Comment d'ailleurs un intérêt aurait-il pu se faire jour puisque, du fait de ces lacunes de l'édition, l'histoire de la mécanique quantique n'existait pas réellement en tant que sujet reconnu de recherches? En témoigne le fait, qui laisse bien des

33. Paris, Alcan, 1933. Il s'agit des mémoires originaux dans lesquels Schrödinger élabore la mécanjque ondulatoire en 1926.

34. Paris, Gauthier-Villars, resp. 1923 et 1928.

35. Paris, Payot, 1929.

36. New York, J. Wiley, 1963.

37. Première édition : Leipzig, S. Hirzel, 1935. Rééd. : 1936, 1942, 1943, 1944, 1947 ,

$1949,1959,1973,1980$. L'édition de 1949 contient dix articles.

38. Munich, R. Piper, 1971.

39. Munich, R. Piper, 1977. Heisenberg est mort en 1976. 
gens incrédules, que le livre de M. Jammer n'a jamais été traduit en France. Il n'y a du reste aujourd'hui encore ni bibliothèque spécialisée, ni revue spécialisée dans le domaine de ce que je suggère d'appeler la «post-modernité dure ». Si bien que les livres n'arrivent pas, ou bien taxés d'une manière qui rappelle la circulation du sel dans la France de l'Ancien Régime. Pour consulter les $A H Q P$, il faut prendre l'avion. Cet état de sous-développement fait qu'à l'étranger la personnalité de la France est en général perçue comme aussi opaque et mystérieuse que celle de Pauli.

On doit à Bernard d'Espagnat d'avoir réanimé de façon spectaculaire l'intérêt pour la philosophie de la physique quantique ${ }^{40}$. Dans le petit nombre des chercheurs qui travaillent actuellement dans ce domaine, chacun lui est redevable au moins d'une orientation, et souvent de sa motivation première. Parmi les philosophes, l'œuvre de Jacques Merleau-Ponty, celle de Jean Largeault ont donné également depuis longtemps la démonstration de l'intérêt de la philosophie de la nature. Enfin, il existe actuellement un séminaire où ces questions sont traitées ${ }^{41}$, et certaines publications des trois dernières années témoignent d'un renouveau évident : ainsi le livre de $M$. Spiro et $G$. Cohen-Tannoudji, ceux de M. Crozon et de M. Paty, la décision du Seuil de publier les Euvres d'Einstein, etc. ${ }^{42}$. L'intendance suivra-t-elle ? Sans bibliothèques ni politique de traduction, la pénurie risque de se maintenir. Le sujet existe, l'intérêt existe, mais les idées ne vivent longtemps que dans des textes.

Catherine Chevalley, C.N.R.S. Paris.

40. Le livre de Bernard D'Espagnat qui a eu le plus d'influence est $A$ la Recherche du réel, Paris, Gauthier-Villars, 1979.

41. Le Séminaire d'épistémologie et histoire de la physique de l'Équipe R.E.H.S.E.I.S., organisé par M. Paty et $\mathbf{O}$. Darrigol.

42. Gilles CoHen-Tannoudi,, Michel SpIro, La Matière-espace-temps, Paris, Fayard, 1986. Michel Crozon, La Matière première, Paris, Le Seuil, 1987. Michel PATY, La Matière dérobée, Paris, Archives contemporaines, 1988. Aussi : Richard FeYnMan, Lumière et matière, trad. Françoise BaliBAR, Alain LAVERne, Paris, Inter éditions, 1987. J. L. HeILBRoN, Planck. Une conscience déchirée, Paris, Belin, 1988. Le livre de John GrIBBIN, Le Chat de Schrödinger, Paris, Éd. du Rocher, 1988, est un bon ouvrage de grande vulgarisation. En 1985, la Revue d'histoire des sciences a consacré son numéro 3-4 en entier à « Bohr et la complémentarité " ". Sur le renouveau des études einsteiniennes, voir la chronique de F. BALIBAR, dans la Revue de synthèse, IV S., 2, 1988, p. 287-296. 